\title{
Optimizing the yield and physico-chemical properties of pine cone cellulose nanocrystals by different hydrolysis time
}

\author{
Daniel García-García • Rafael Balart • Juan Lopez-Martinez • \\ Monica Ek • Rosana Moriana
}

Received: 15 December 2017/ Accepted: 15 March 2018/Published online: 23 March 2018

(C) The Author(s) 2018

\begin{abstract}
Cellulose nanocrystals (CNCs) were isolated for the first time from pine cones (PC) by alkali and bleaching treatments and subsequent sulfuric acid hydrolysis $(64 \%)$ at $45^{\circ} \mathrm{C}$. The influence of the hydrolytic reaction time $(30,45$, and $90 \mathrm{~min})$ on the yield, chemical composition and structure, and thermal stability of CNCs was evaluated. The removal of non-cellulosic constituents during the alkaline and bleaching treatment resulted in high pure cellulosic fibres. The isolation of CNCs from these cellulosic fibres at different reaction times was verified by the nano-dimensions of the individual crystals $(<3$ and $<335 \mathrm{~nm}$ of average diameter and length, respectively). The highest yield $(15 \%)$ and the optimum CNCs properties in terms of aspect ratio, thermal stability and crystallinity were obtained for an
\end{abstract}

D. García-García · R. Balart · J. Lopez-Martinez Instituto de Tecnología de Materiales (ITM), Universitat Politècnica de València (UPV), Plaza Ferrándiz y

Carbonell 1, 03801 Alcoy, Alicante, Spain

M. Ek $\cdot$ R. Moriana $(\bowtie)$

School of Engineering Sciences in Chemistry, Biotechnology and Health, Department of Fibre and Polymer Technology, KTH-Royal Institute of Technology, 10044 Stockholm, Sweden e-mail: rosana@kth.se;

rosana.moriana.torro@his.se

R. Moriana

School of Engineering Science, HIS-University of Skövde, Skövde, Högskolevägen, 54128 Skövde, Sweden extraction time of $45 \mathrm{~min}$. PC appeared to be a new promising source of cellulose fibres and CNCs with potential to be applied as reinforcement in composites and for food-packaging.

Keywords Pine cones - Cellulose nanocrystals · Sulfuric hydrolysis conditions · Physico-chemical properties · Yield-recovery

\section{Introduction}

During the last years, concerns about sustainable development have increased significantly leading to a major interest for the research and development of environmentally friendly materials as an alternative to petroleum-based materials. Cellulose is the most abundant biopolymer on earth and is characterized by being renewable, biodegradable and non-toxic (Haafiz et al. 2013; Peng et al. 2011). Cellulose consists of linear homopolysaccharide chains of $\beta$-Dglucopyranose units linked together by $\beta$-1,4-glycosidic bonds (Luzi et al. 2016). Intramolecular and intermolecular hydrogen bonds are established between the cellulosic chains resulting in ordered/packed crystalline structures which are intercalated with amorphous regions in the fibril structure (Jiang and Hsieh 2013; Mariano et al. 2014; Sheltami et al. 2012; Siqueira et al. 2010). These crystalline domains can be separated from each other by overcoming the 
extensive and strong inter-fibrillar hydrogen bonds with acid treatment (Camarero Espinosa et al. 2013; Lamaming et al. 2015; Lu and Hsieh 2012), specific enzymes (Satyamurthy et al. 2011) and/or intense mechanical forces (Chen et al. 2011; Deepa et al. 2011). Among these extractive methods, the use of sulfuric acid to provoke the cleavage of the glycosidic bonds, achieving the disintegration of the cellulose amorphous region, has been the most widely used (Lu et al. 2013; Mariano et al. 2014; Neto et al. 2013). Cellulose nanocrystals (CNCs) obtained from sulfuric acid hydrolysis have been of great scientific interest due to their high crystallinity, low density, rod-like shape, high aspect ratio (diameter/length), high specific surface area, good mechanical properties (high stiffness and elastic modulus), low coefficient of thermal expansion, stability in aggressive media, gas permeability and optical transparency (Ioelovich 2012; Jiang and Hsieh 2013; Mueller et al. 2014; Peng et al. 2011). Most of these CNCs properties (such as aspect ratio, morphology, thermal stability and degree of crystallinity) are highly dependent on the hydrolysis conditions as well as on the raw cellulosic material they are extracted from Le Normand et al. (2014). The reaction time has been identified as one of the most important parameters to consider during the CNCs extraction. Kargarzadeh et al. (2012) obtained CNCs from kenaf with different hydrolysis reaction times (20, 30, 40, 60, 90, and $120 \mathrm{~min}$ ) and specific thermal and crystallinity properties were achieved for each time. Silvéiro et al. (2013) also studied the effect of the acid hydrolysis time (30,60, and $90 \mathrm{~min}$ ) on the chemical, physical and thermal properties of CNCs from corncob and the highest crystallinity and thermal stability were obtained with an hydrolytic time of $60 \mathrm{~min}$. The origin of the cellulose raw materials on the particular performance of CNCs has been also evaluated through different comparative studies, where the hydrolysis conditions were fixed. CNCs were obtained from a variety of sources; however, most research efforts have been focused on partially purified versions of woods (such as microcrystalline cellulose and bleached pulp). Beck-Candanedo et al. (2005) investigated the suspension properties of CNC obtained by hydrolysis of a softwood (spruce) and a hardwood (eucalyptus) pulp. These two suspensions had similar CNCs dimensions and surface charge, implying that the basic unit of wood cellulose organization is the same for the two species. During the last decade, the use of residual lignocellulosic biomass from agriculture, food and forest to produce CNCs has become impellent due to the increasing demand of finding cheaper sources and higher extraction yields alternatives to produce nanocelluloses as reinforcing agents for composites (Maiti et al. 2013). Deepa et al. (2015) obtained CNCs from various biomass residues (sisal, kapok, banana rachis, pineapple leaf and coir) and differences in their size, crystallinity and thermal stability were detected depending on the used raw material. In previous studies, bark, pine needles, branches and woody chips were proposed as new cost-effective forest raw materials to isolate CNCs. The feasibility of obtaining CNCs with specific performances depending on the physico-chemical properties of the cellulose fibres in the forest residues was proved (Le Normand et al. 2014; Moriana et al. 2016). A comparative study of the aspect ratio, crystallinity and thermal stability of the extracted $\mathrm{CNCs}$ was proposed to assess their performance as reinforcing agent in composites (Moriana et al. 2016).

In this paper, we investigate for the first time the feasibility of using pine cones from Pinus Pinea (Stone pine) as a raw material to produce CNCs. Pinus Pinea (Stone pine) is one of the most important species in the Mediterranean region due mainly to the production of pine nuts, a culinary ingredient with an increasing demand (Özgüven and Vursavuş 2005). Nowadays, Spain, Portugal and Italy are the countries with the largest population of this tree species with 490,000; 175,000 and 46,000 ha respectively (Loewe et al. 2017). After collecting the pine nuts the cones are discarded, leading to a high amount of residue, which has no industrial or economic value. Currently, small amounts of pine cones are being used as home fuel, however most of this waste ends up being incinerated or thrown into the field creating a risk of fire (Almendros et al. 2015). The study and research of alternatives for the recovery of this food-waste by obtaining value-added products will contribute to move towards a circular bio-based economy and to develop a biorefinery concept with environmental, social and economic benefits ( $\mathrm{Li}$ et al. 2016). The goals of this study are: to evaluate the feasibility of isolating CNCs from pine cones using different hydrolytic times $(30,45$, and $90 \mathrm{~min})$; to determine the overall recovery yield, the physico-chemical properties and thermal behavior of the produced 
$\mathrm{CNCs}$; and, to estimate the optimal processing conditions to obtain CNCs suitable for being used as reinforcements in polymeric-based composites.

\section{Materials and methods}

\section{Materials}

Pine cones (PCs) were collected from a Pine forest (Pinus Pinea) in Alicante area (Spain). These forest residues were conditioned at $40{ }^{\circ} \mathrm{C}$ during 1 week. The raw PCs were ground in a Wiley mill (Thomas Scientific, USA) to pass through a $20 \mu \mathrm{m}$ mesh screen. Analytical grade chemicals used for the experimental procedure were: sodium hydroxide $(\mathrm{NaOH} 99 \%)$, sulfuric acid $\left(\mathrm{H}_{2} \mathrm{SO}_{4} 98 \%\right)$, sodium chlorite $\left(\mathrm{NaClO}_{2}\right.$ $80 \%)$, sodium acetate $\left(\mathrm{C}_{2} \mathrm{H}_{3} \mathrm{NaO}_{2}>99 \%\right)$ and glacial acetic acid $\left(\mathrm{CH}_{3} \mathrm{COOH} 99.7 \%\right)$. All of them were purchased from Sigma-Aldrich (Sigma-Aldrich, Germany). All water used was purified by Milli-Q water (Millipore Corporate, USA). Polysaccharide standards (cellulose, starch, galactomannan, glucomannan, arabinoxylan, arabinogalactan, arabinan) for the chemical analysis were purchased from Sigma-Aldrich or Megazyme (Megazyme, Ireland).

\section{Isolation of cellulose nanocrystals (CNCs)}

The isolation of the CNCs was achieved by subjecting milled PC samples to different chemical treatments. First, an alkaline and bleaching treatment was proposed to remove the extractives, lignin and hemicelluloses and to produce the subsequent isolation of the cellulose fibres from the raw material. Second, a hydrolytic treatment with sulfuric acid was performed to remove the amorphous regions to obtain the CNCs.

\section{Alkaline and bleaching treatment}

Milled PC samples were subjected to an alkaline and bleaching treatment following the same methodology previously described by Moriana et al. (2016). Three different batches of PC (4\% w/v) were treated with sodium hydroxide solution $(4.5 \% \mathrm{w} / \mathrm{v} \mathrm{NaOH})$ for $2 \mathrm{~h}$ at $80{ }^{\circ} \mathrm{C}$ under vigorous mechanical stirring. This alkaline treatment was repeated three times and after each treatment, the resulting material was washed with water until the removal of the chemicals and subsequently dried at room temperature overnight. After alkali treatment, three different batches (4\% w/v) of alkaline pine cones (APC) were bleached with a solution made up of equal parts (1:1:1) of $1.7 \mathrm{wt} \%$ aqueous sodium chlorite, acetate buffer $(0.2 \mathrm{M}, \mathrm{pH}$ 4.8 ), and water. The bleaching procedure was performed at $80{ }^{\circ} \mathrm{C}$ for $4 \mathrm{~h}$ and repeated five times. After each bleaching treatment the bleached pine cones (BPC) were filtered and washed with Milli-Q water and dried at room temperature for $24 \mathrm{~h}$.

\section{Acid hydrolysis treatment}

The acid hydrolysis treatment was performed on the BPC. BPC were dried at $40{ }^{\circ} \mathrm{C}$ for $24 \mathrm{~h}$ in an aircirculating oven and subsequently grinded in a Wiley Mill (Thomas Scientific, New Jersey, USA) using a $20 \mu \mathrm{m}$ mesh screen. The milled BPC were hydrolyzed in sulfuric acid solution (65\% wt) at $45{ }^{\circ} \mathrm{C}$ for 30,45 , and 90 min under mechanical stirring. At least three different batches at $4 \% \mathrm{w} / \mathrm{v}$ of BPC were prepared for each hydrolyzed time. The suspensions were diluted with ice cubes to stop the hydrolysis reaction. Afterwards, they were washed with Milli-Q water by successive centrifugations in an Avanti J-E centrifuge (Beckman Coulter, California, USA) at 13,000 rpm for $10 \mathrm{~min}$ at $4{ }^{\circ} \mathrm{C}$ until the supernatant reached a constant $\mathrm{pH}$ of 5 . After, the suspensions were dialyzed with purified water during 1 week until the neutral $\mathrm{pH}$. Finally, the suspensions were sonicated for $5 \mathrm{~min}$ at an amplitude of $27 \%$ using an ultrasonic homogenizer Vibra-cell Mod. VCX 750 (Sonics and Materials Inc., Connecticut, USA) in an ice bath to avoid overheating. To remove the largest particles, the suspensions were centrifuged and the sediments were discharged. The $\mathrm{CNC}$ suspensions were stored at $4{ }^{\circ} \mathrm{C}$ for further characterization. The obtained $\mathrm{CNCs}$ suspensions were labelled depending on the extraction time as follows: $\mathrm{CNC}_{30}, \mathrm{CNC}_{45}$ and $\mathrm{CNC}_{90}$, for the $\mathrm{CNC}$ suspensions extracted at 30, 45, and $90 \mathrm{~min}$ respectively.

Characterization of alkaline, bleached and hydrolyzed PC samples

\section{Gravimetric analysis}

The gravimetric yield of each experimental treatment (alkaline, bleaching and hydrolysis) was determined 
by weighing the dried samples before and after of each experimental procedure. At least three different replications of each sample and batch were considered to calculate the average and the standard deviations.

\section{Chemical composition analysis}

The chemical composition of all the studied samples (PC, APC, BPC, $\mathrm{CNC}_{30}, \mathrm{CNC}_{45}$ and $\mathrm{CNC}_{90}$ ) was evaluated following the methodology previously described by Moriana et al. (2015). The dry matter of samples was determined in a Mettler Toledo HB43 moisture analyzer (Mettler Toledo SAE, USA). The ash content was determined gravimetrically after heating the dry samples in a furnace at $525^{\circ} \mathrm{C}$ for $6 \mathrm{~h}$, following the TAPPI standard method T211 om02. The determination of acid insoluble (Klason) lignin in the samples was carried out according to the TAPPI T222. The total amount of soluble extractives in the PC was determined by sequential extraction with ethanol, toluene and hot water (de Carvalho et al. 2015). The carbohydrates composition was assessed by conventional two-step Saeman hydrolysis (Saeman et al. 1954), followed by quantification of the released monosaccharides using high-pH anion-exchange chromatography with pulsed amperometric detection (HPAEC-PAD). In short, $1 \mathrm{mg}$ of dry sample was initially pre-hydrolyzed with $250 \mu \mathrm{L} 72 \% \mathrm{H}_{2} \mathrm{SO}_{4}$ at room temperature for $3 \mathrm{~h}$, diluted until a final concentration of $1 \mathrm{M} \mathrm{H}_{2} \mathrm{SO}_{4}$, and then subjected to a second hydrolysis step at $100{ }^{\circ} \mathrm{C}$ for $3 \mathrm{~h}$. The hydrolysates were centrifuged at $4{ }^{\circ} \mathrm{C}$ and subjected to analysis directly without any other purification or neutralization. The released monosaccharides were separated on a Dionex ICS3000 system (Dionex Corporation, California, USA) by $10 \mu \mathrm{L}$ injection of the filtered hydrolysates on a Dionex Carbopac PA1 column at $30{ }^{\circ} \mathrm{C}$ at a flow rate of $1 \mathrm{~mL} \mathrm{~min}{ }^{-1}$. Neutral sugars (glucose, mannose, xylose, arabinose, galactose, and rhamnose) and uronic acids (galacturonic and glucuronic acid) were analyzed separately using different elution profiles (Wright and Wallis 1996). The samples were analyzed in triplicate.

\section{Scanning electron microscopy (SEM)}

The morphologies of PC, APC and BPC were evaluated using a scanning electron microscopy Phenom (FEI Company, Eindhoven, the Netherlands) operated at $5 \mathrm{kV}$ acceleration voltage. Prior the analysis the samples were coated with a thin layer of gold/palladium alloy in a sputter coater EMITECH model SC7620 (Quorum Technologies Ltd., East Sussex, UK).

\section{Atomic force microscopy (AFM)}

AFM imaging of CNCs with different hydrolysis times was performed in a Nanoscope IIIa Multimode scanning probe microscope (Digital Instruments Inc., New York, USA). A few drops of diluted nanocrystals suspension were deposited onto a freshly cleaved mica surface and air-dried. All images were obtained using the tapping-mode in air at room temperature with RTESP silica cantilevers (Bruker) having a tip with a radius of $8 \mathrm{~nm}$ and a spring constant of $20-80 \mathrm{~N} \mathrm{~m}^{-1}$ and resonance frequencies between 306 and $366 \mathrm{kHz}$. Lengths and diameters were obtained from printouts of several height mode AFM images, using the section analysis tool of the NanoScope Analysis software Version 1.40. AFM samples from each type of CNCs were prepared from the three different batches to evaluate the reproducibility of the experimental procedure. No significant differences in the CNC dimensions were detected from batch to batch. Therefore, more than a hundred $\mathrm{CNCs}$ of each material were randomly selected and measured to determine their average length and diameter.

\section{Thermogravimetric analysis (TGA)}

The thermal behavior of PC, APC, BPC and the different CNCs was evaluated by thermogravimetric analysis (TGA) (Le Normand et al. 2014). The samples, with an average mass of $4.5 \mathrm{mg}$, were placed in an alumina crucible $(70 \mu \mathrm{L})$ and heated from 25 to $750{ }^{\circ} \mathrm{C}$ at a constant heating rate of $10{ }^{\circ} \mathrm{C} \mathrm{min}^{-1}$ on a Mettler-Toledo TGA/DSC 1 thermobalance (Schwerzenbach, Switzerland) under nitrogen atmosphere $\left(50 \mathrm{~mL} \mathrm{~min}{ }^{-1}\right)$. The onset temperature, maximum decomposition temperature and mass loss percentages, for each thermal decomposition process were obtained using the STAR $^{\mathrm{e}}$ Evaluation Software. At least three replicates of each sample were performed and evaluated. 
$X$-ray diffraction spectroscopy (XRD)

X-ray diffraction of all the studied samples was obtained using a Bruker CCD-Apex apparatus equipped with a $\mathrm{X}$-ray generator ( $\mathrm{Ni}$ filtered $\mathrm{Cu}-\mathrm{K} \alpha$ radiation) operated at $40 \mathrm{kV}$ and $40 \mathrm{~mA}$ with $2 \theta$ varying from $10^{\circ}$ to $60^{\circ}$ at a scan rate of $2^{\circ} \mathrm{min}^{-1}$. The measurements were repeated at least twice for each sample. The crystalline index $(\mathrm{CrI})$ of the PC before and after the different treatments and the CNCs with different hydrolysis times was determined by referring to diffraction intensity of crystalline and amorphous regions using the empirical method proposed by Segal et al. (1959):

$$
C r I=\frac{I_{200}-I_{a m}}{I_{200}} \times 100
$$

where $I_{200}$ is the maximum intensity at plane 200 located at a diffraction angle around $2 \theta=22^{\circ}$, and $I_{\mathrm{am}}$ stands for the minimum intensity from the amorphous part of the sample, measured approximately at a diffraction angle of around $2 \theta=18^{\circ}$.

\section{Fourier transform infrared spectrometry (FTIR)}

FTIR spectra of all the samples were collected at room temperature on a Spectrum 2000 FTIR spectrometer from Perkin-Elmer (Perkin-Elmer Inc., Massachusetts, USA) equipped with a golden singlereflection accessory for ATR measurements. Each spectrum was obtained as 24 individual scans at $4 \mathrm{~cm}^{-1}$ resolution in the wavenumber range comprised between 4000 and $600 \mathrm{~cm}^{-1}$. The measurements were repeated three times for each sample. The spectra were automatically baseline-corrected and smoothened using Omnic 7 Software.

\section{Results and discussion}

Visual description and gravimetric yield of the isolation procedure

The visual appearance of the PC samples during the entire isolation process together with the gravimetric yields obtained after each chemical treatment are shown in Fig. 1. The removal of $35 \%$ of the initial PC sample mass during the alkaline treatment, resulted in a sample with a lighter brown appearance, indicating the partially removal of lignin, pectins and hemicelluloses (Neto et al. 2013). During the bleaching treatment, $26 \%$ of mass was lost and the obtained white fibrous sample indicated most of the lignin was removed and the cellulose fibres were isolated (dos Santos et al. 2013). The acid hydrolysis of the bleached samples resulted in a blurry suspension with a gel-like behavior. Similar yields were obtained for the $\mathrm{CNC}_{30}$ and $\mathrm{CNC}_{45}$ samples, whereas the $\mathrm{CNC}_{90}$ had the lowest yield. During the second centrifugation step, different amounts of cellulosic solid residues (CSR) were precipitated for each hydrolytic time (7.5, 1 , and $2 \%$ for $\mathrm{CNC}_{30}, \mathrm{CNC}_{45}$ and $\mathrm{CNC}_{90}$, respectively). The higher CSR obtained for the $\mathrm{CNC}_{30}$ indicates higher agglomerates and less homogenous hydrolysis was produced due to an insufficient hydrolytic time to uniformly remove the amorphous domains of the bleached samples. On the other hand, the similar content of CSR obtained at 45 and $90 \mathrm{~min}$ and the lowest yield value for the $\mathrm{CNC}_{90}$ suggests a partial degradation of the crystalline domains. The higher overall yield was therefore for the $\mathrm{CNC}_{45}$ with a value of $14.7 \%$. Similar yields were published for CNCs extracted from branches, pine-needles, rachis of date palms tree and capim dourado under similar isolation conditions (Bendahou et al. 2009; Moriana et al. 2016; Siqueira et al. 2010).

The effect of the specific hydrolysis times on the chemical composition, morphology, crystallinity and thermal stability of the resulting CNCs was evaluated to assess the most suitable conditions to produce CNCs to be used as reinforcing agents for polymer matrices in composites.

\section{Chemical composition}

The identification of the relative amount of each component present in each stage of the isolation process was performed by the combined results from the carbohydrate composition, the ash content, extractives estimation and lignin Klason determination (Table 1). The carbohydrate composition was determined from the quantification of the area peaks from chromatographic curves corresponding to the glucose, mannose, xylose, arabinose galactose, rhamnose, galacturonic and glucuronic acid of each sample. The amount of cellulose results from the percentage of glucose, whereas the amount of hemicellulose/pectin (such as mannans, xylans, arabinogalactans, 


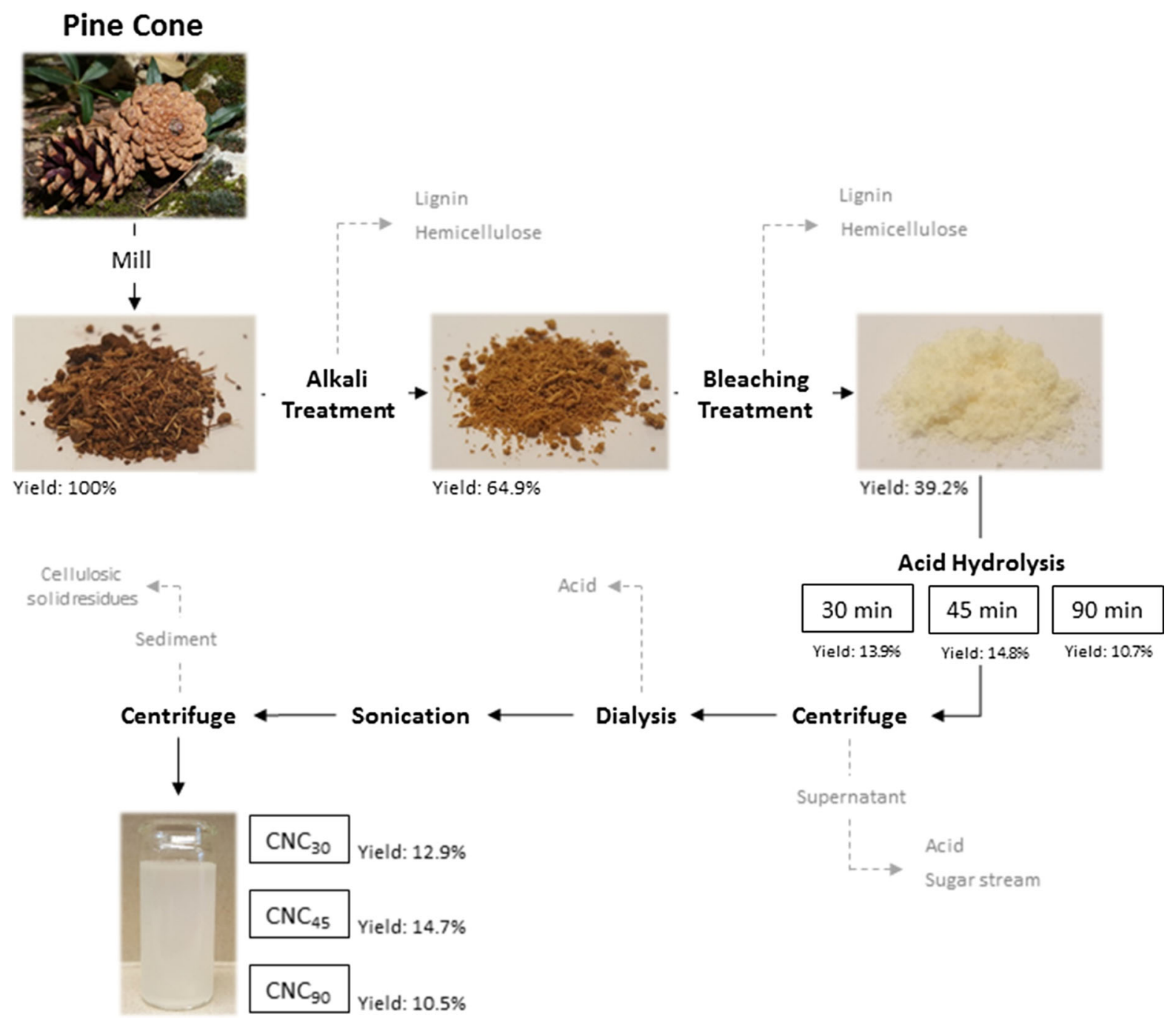

Fig. 1 Schematic representation of the pine cone CNC isolation procedure with different hydrolysis times

galacturonan) corresponds to the percentage of the remaining sugars. Some hemicelluloses may contain as well glucose monomers in their structure (e.g. glucomannans) but their contribution is somewhat minor compared to cellulose, as it can be observed in the low mannose percentage shown in the Table 1. The PC samples show a high amount of glucose (45.3\%) and lignin in their composition (39.6\%). The hemicellulose/pectin content can be primarily assigned to galactoglucomannan (with a $2.9 \%$ of mannose and $1.3 \%$ of galactose) which is the main hemicellulose present in softwoods (Willför et al. 2005). The chemical composition of PC showed a higher cellulose content compared to other forest residues that were previously proposed as raw materials to obtain CNCs such as woody chips, pine needles, branches or bark spruce (Le Normand et al. 2014; Moriana et al. 2015). During the alkali treatment the lignin and hemicellulose/pectin content was reduced. However, the most significant reduction of lignin took place during the bleaching step reaching a content of $4.1 \%$. On the other hand, the hemicellulose/ pectin content did not decrease due to the bleaching process $(4.4 \%)$, but the reached value was still lower than those from other forest residues at this stage (21\% for the branches and pine-needles) (Le Normand et al. 2014; Moriana et al. 2015). These low values of both lignin and hemicellulose/pectin resulted in BPC 
Table 1 Yield obtained after each chemical treatment and chemical composition of PC, APC, BPC and CNCs with different hydrolysis times

\begin{tabular}{|c|c|c|c|c|c|c|}
\hline Treatment & $\mathrm{PC}$ & APC & $\mathrm{BPC}$ & $\mathrm{CNC}_{30}$ & $\mathrm{CNC}_{45}$ & $\mathrm{CNC}_{90}$ \\
\hline Yield $^{\mathrm{a}}$ & 100 & $64.9 \pm 3.5$ & $60.3 \pm 1.5$ & $32.7 \pm 0.4$ & $37.1 \pm 0.4$ & $26.8 \pm 0.3$ \\
\hline Arabinose & $0.23 \pm 0.01$ & $0.20 \pm 0.00$ & $0.11 \pm 0.01$ & $<0.1$ & $<0.1$ & $<0.1$ \\
\hline Rhamnose & $<0.1$ & $<0.1$ & $<0.1$ & $<0.1$ & $<0.1$ & $<0.1$ \\
\hline Galactose & $1.31 \pm 0.03$ & $1.10 \pm 0.00$ & $0.88 \pm 0.07$ & $<0.1$ & $<0.1$ & $<0.1$ \\
\hline Glucose & $45.33 \pm 0.04$ & $62.62 \pm 0.02$ & $88.99 \pm 0.14$ & $95.99 \pm 0.07$ & $95.65 \pm 0.01$ & $97.85 \pm 0.05$ \\
\hline Xylose & $0.23 \pm 0.01$ & $0.14 \pm 0.02$ & $0.11 \pm 0.00$ & $0.16 \pm 0.03$ & $0.23 \pm 0.01$ & $<0.1$ \\
\hline Mannose & $2.91 \pm 0.05$ & $2.34 \pm 0.03$ & $2.76 \pm 0.22$ & $2.40 \pm 0.04$ & $2.50 \pm 0.00$ & $0.50 \pm 0.02$ \\
\hline Galacturonic acid & $0.39 \pm 0.08$ & $0.13 \pm 0.04$ & $0.21 \pm 0.07$ & $<0.1$ & $<0.1$ & $<0.1$ \\
\hline Glucuronic acid & $0.30 \pm 0.07$ & $0.18 \pm 0.03$ & $0.30 \pm 0.09$ & $0.18 \pm 0.02$ & $0.17 \pm 0.01$ & $<0.1$ \\
\hline Total carbohydrates & $50.71 \pm 0.30$ & $66.70 \pm 0.13$ & $93.36 \pm 0.61$ & $98.86 \pm 0.17$ & $98.66 \pm 0.03$ & $98.47 \pm 0.10$ \\
\hline Cellulose & $45.33 \pm 0.04$ & $62.62 \pm 0.02$ & $88.99 \pm 0.14$ & $95.99 \pm 0.07$ & $95.65 \pm 0.01$ & $97.85 \pm 0.05$ \\
\hline Hemicellulose/pectin & $5.38 \pm 0.25$ & $4.08 \pm 0.12$ & $4.37 \pm 0.47$ & $2.87 \pm 0.09$ & $3.01 \pm 0.02$ & $0.62 \pm 0.02$ \\
\hline Extractives & $6.51 \pm 0.31$ & - & - & - & - & - \\
\hline Klason lignin & $39.66 \pm 0.01$ & $29.76 \pm 0.02$ & $4.10 \pm 0.06$ & N/A & N/A & N/A \\
\hline Ash & $3.12 \pm 0.25$ & $3.54 \pm 0.23$ & $2.54 \pm 0.32$ & $1.14 \pm 0.04$ & $1.34 \pm 0.04$ & $1.53 \pm 0.05$ \\
\hline
\end{tabular}

${ }^{\text {a }}$ The gravimetric yields for each treatment were calculated based on the total dry weight (100\%) of the previous treatment

samples with an extraordinary high cellulose content (93.4\%), showing their potential to be used as raw materials to isolate $\mathrm{CNCs}$.

During the hydrolytic treatment is expected hemicelluloses and pectins hydrolyzed together with the amorphous part of the cellulose and became soluble (Moriana et al. 2015). However, it was observed that the mannose content in $\mathrm{CNC}_{30}$ and $\mathrm{CNC}_{45}$ was similar to that present in the BPC sample. This is because the mannose is more resistant to hydrolysis and dissolution during the preparation of the CNCs than other amorphous cell wall components (Hannuksela et al. 2002; Iwata et al. 1998). However, in the $\mathrm{CNC}_{90}$ the mannose content significantly decreases, thus indicating greater removal of the hemicellulose content and, maybe, the degradation of the cellulose. This fact can also be pointed out due to the small gravimetric yield (Table 1) of the $\mathrm{CNC}_{90}$ in comparison to $\mathrm{CNC}_{45}$. The $\mathrm{CNC}$ yields for $\mathrm{CNC}_{30}$ and $\mathrm{CNC}_{90}$ (32.7 and $26.8 \%$ respectively) are similar to those reported by Le Normand et al. (2014) for spruce bark and by Brito et al. (2012) for bamboo fibres. However, the $\mathrm{CNC}_{45}$ yields are higher and more similar to those reported by Moriana et al. (2016) for woody chips.

\section{Morphological surface}

SEM and AFM images were used to follow the morphological surface changes in materials during the entire CNC isolation process. Figure 2 shows the SEM micrographs of the raw PC (Fig. 2a), after alkali (Fig. 2b) and bleaching treatment (Fig. 2c). PC is composed of oriented fibre bundles that are bonded together by a large amount of amorphous material, many irregularities and impurities are also seen on the surface. After the alkaline treatment, a greater definition of the cellulosic fibres was observed (Fig. 2b). The non-cellulosic components of the PC that act as binders of the cellulosic fibres, such as hemicellulose, lignin, pectin and other impurities were partially removed, achieving an opening of the matrix that will enhance the penetration of the bleaching agents during the subsequent treatment (Johar et al. 2012). After bleaching, most of lignin present in the PC was removed; chlorine is able to oxidize lignin, giving rise to the formation of hydroxyl, carbonyl and carboxylic groups, which allow lignin solubilization (Cherian et al. 2010). Removal of most lignin and hemicellulose increased the defibrillation, resulting in a large amount of individual cellulosic fibres, with an average diameter of $24.2 \pm 4.5 \mu \mathrm{m}$ (Fig. 2c). These values are 

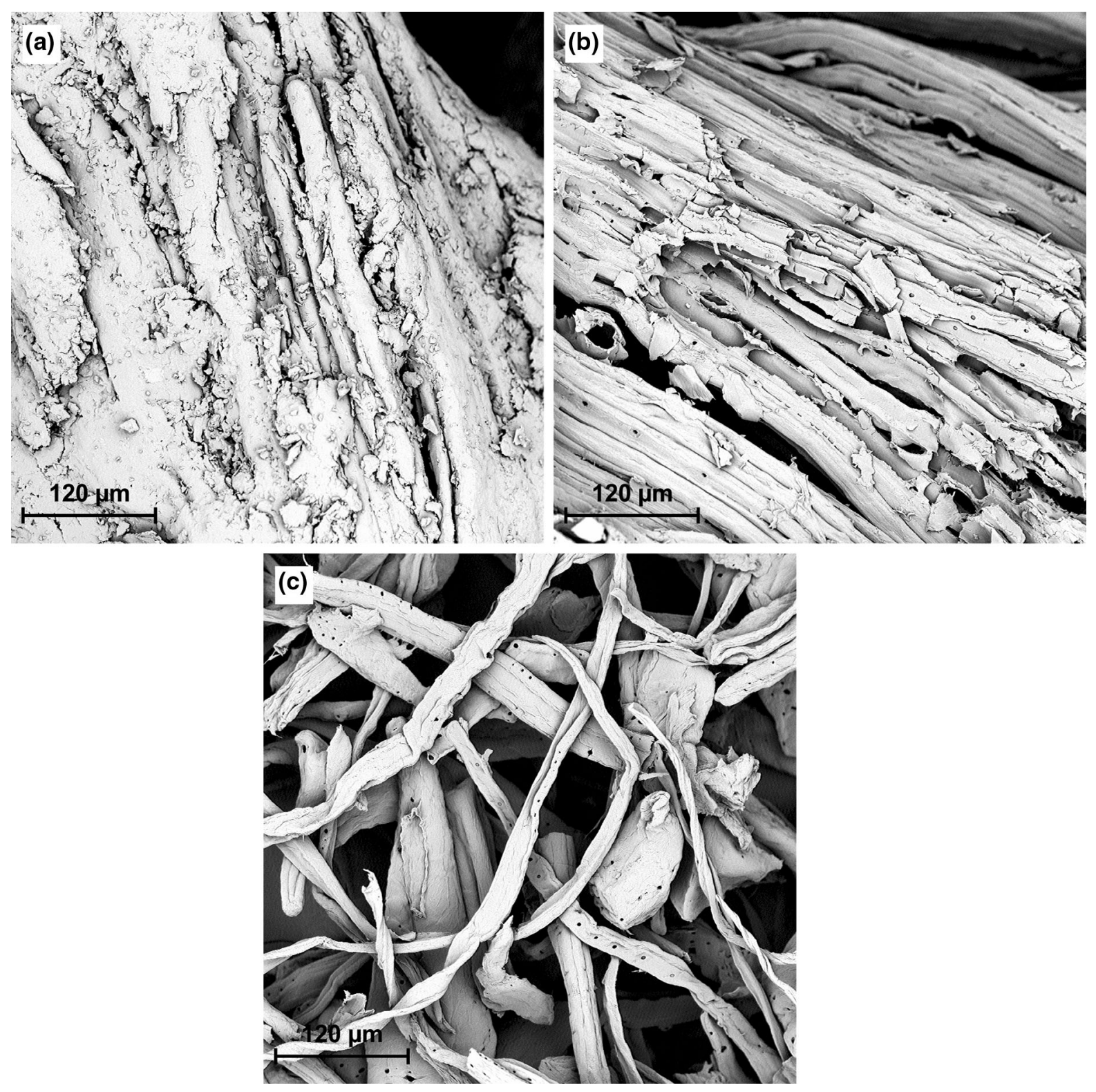

Fig. 2 SEM micrographs of a raw pine cone (PC), b alkaline (APC) and $\mathbf{c}$ bleached (BPC) samples

similar to the average width observed for other forest residues, such as woody chips (around $23.5 \mu \mathrm{m}$ ) and branches (around $19.6 \mu \mathrm{m}$ ) (Moriana et al. 2016).

The morphology and size distribution of CNCs with different hydrolysis times were studied by AFM. Figure 3 shows an example of AFM images of the isolated $\mathrm{CNC}_{45}$, as well as the histograms of each CNCs, with the corresponding length (L) and width (D) distribution. The obtained PC nanocrystals had a rod-like aspect. Nevertheless, some aggregates between them can be observed. This is mainly due to the strong hydrogen bonds established between crystals (Le Normand et al. 2014). The highest diameter dispersion was for the $\mathrm{CNC}_{45}$ with a diameter ranging from 1.8 to $5.7 \mathrm{~nm}$. These dimensions are typical values for CNCs lengths from wood (Beck-Candanedo et al. 2005). On the other hand, the $\mathrm{CNC}_{90}$ showed the lowest length dispersion (from 106.7 to $410 \mathrm{~nm}$ ). These dimensions are similar to typical length values for wood CNC (100-400 nm) (Beck-Candanedo et al. 
(a)

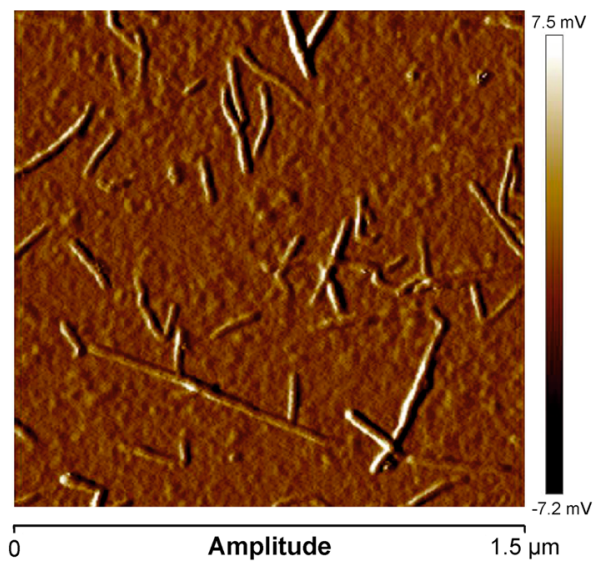

(c)

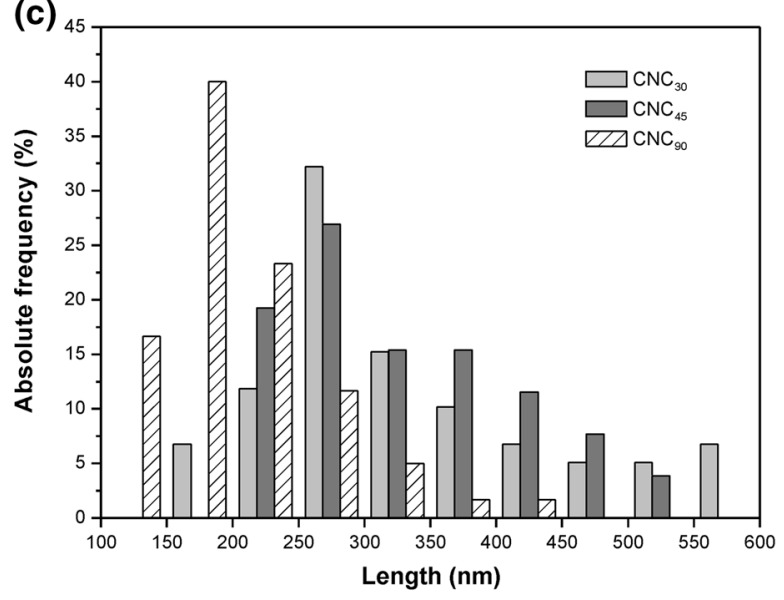

(b)

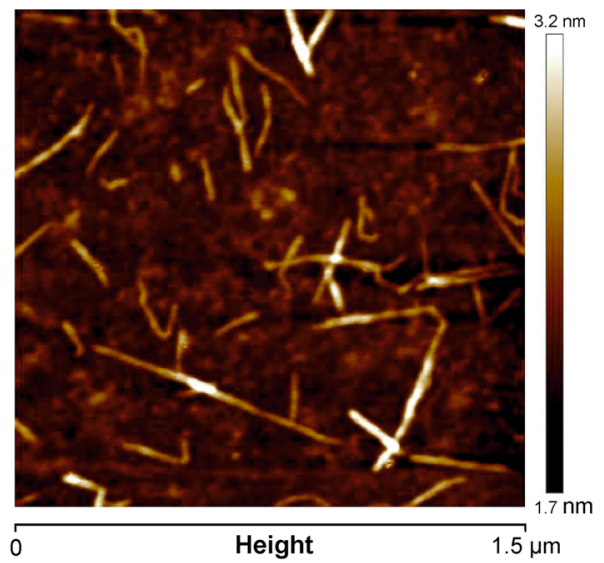

(d)

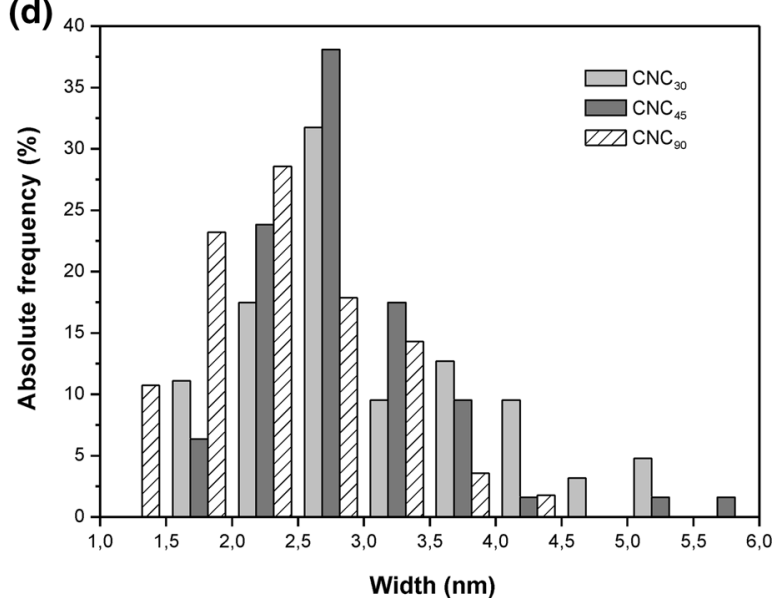

Fig. 3 AFM images of $\mathrm{CNC}_{45}$ in a amplitude, and $\mathbf{b}$ height mode (scale bar $1.5 \mu \mathrm{m}$ ). Size distribution histograms of the $\mathrm{CNCs}$ at different hydrolysis time: $\mathbf{c}$ length and $\mathbf{d}$ width

2005; Bondeson et al. 2006). The obtained nanoparticles were characterized by their average length and width and their limit values (maximum and minimum) together with their aspect ratio (L/D) (Table 2). The average dimensions of the $\mathrm{CNC}_{30}$ and $\mathrm{CNC}_{45}$ were very similar between them $(330 \mathrm{~nm}$ of length and $3 \mathrm{~nm}$ of width), which is very similar in diameter to other forest residues (branches and bark) (Le Normand et al. 2014; Moriana et al. 2016), but much higher in length. For longer hydrolysis times (90 min), a considerable decrease in CNC size $(206.5 \mathrm{~nm}$ of length and $2.4 \mathrm{~nm}$ of width) was detected. This might be due to the partial destruction of the crystalline domain due to the long hydrolysis time (Neto et al. 2013). The CNCs aspect ratio is one of the most important parameters in determining their reinforcing capacity for nanocomposite applications. Nanocrystals with a high aspect ratio can provide higher reinforcement effect, leading to an increase in mechanical and thermal properties at low loadings
Table 2 Length, width and aspect ratio of CNCs with different hydrolysis times obtained by AFM analysis

\begin{tabular}{lllllllll}
\hline Sample & \multicolumn{2}{l}{ Length L $(\mathrm{nm})$} & & \multicolumn{3}{l}{ Width D $(\mathrm{nm})$} & \multicolumn{2}{c}{ Average aspect ratio (L/D) } \\
\cline { 2 - 3 } & Max & Min & Average & & Max & Min & Average & \\
\hline $\mathrm{CNC}_{30}$ & 572.3 & 165.6 & $334.9 \pm 112.3$ & & 5.5 & 1.6 & $3.0 \pm 0.9$ & 111.6 \\
$\mathrm{CNC}_{45}$ & 501.2 & 223.4 & $328.9 \pm 82.2$ & & 5.7 & 1.8 & $2.9 \pm 0.7$ & 113.4 \\
$\mathrm{CNC}_{90}$ & 410.0 & 106.7 & $206.5 \pm 64.5$ & & 4.4 & 1.2 & $2.4 \pm 0.7$ & 86.0 \\
\hline
\end{tabular}


(Eichhorn et al. 2010). In this case, $\mathrm{CNC}_{30}$ and $\mathrm{CNC}_{45}$ offer a greater reinforcement potential than the $\mathrm{CNC}_{90}$ in nanocomposite applications. PC nanocrystals have a higher aspect ratio than those CNCs obtained from other agro-forestry residues under similar conditions, such as woody chips, branches, pine needles (Moriana et al. 2016), spruce bark (Le Normand et al. 2014), black spruce (Beck-Candanedo et al. 2005), Pandanus tectorius (Sheltami et al. 2012), rice straw (Lu and Hsieh 2012), rice husk (Johar et al. 2012) and soy hull (Neto et al. 2013).

Crystallinity and crystal size

The X-ray method was used to characterize the crystalline structure changes in the samples during the entire isolation process and to assess the differences due to the hydrolysis time. Figure 4 shows the $\mathrm{X}$-ray patterns of PC at different stages of treatment. As it can be seen, all the patterns are characterized by two peaks around $2 \theta=16.3^{\circ}$ and $22.5^{\circ}$, typical of the crystalline structure of cellulose I, and an amorphous broad hump $\left(2 \theta=18-19^{\circ}\right)$. After the chemical treatments the peak around $22.5^{\circ}$ increased and became more defined. This indicates a higher crystallinity degree due to the partial removal of the amorphous regions (Sheltami et al. 2012).

During the alkaline and bleaching treatments, an increment in the CrI was observed (from 42.6 to 65\%) due to the reduction and removal of amorphous noncellulosic compounds such as lignin and hemicellulose (Chen et al. 2011; Rosli et al. 2013). During the

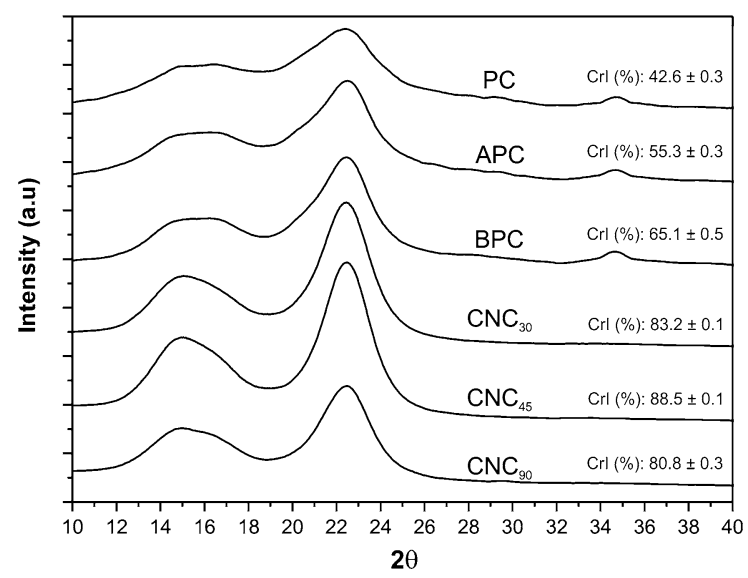

Fig. 4 X-ray diffraction, Crystallinity Index (CrI) of PC, APC, $\mathrm{BPC}$ and $\mathrm{CNCs}$ with different hydrolysis times hydrolysis treatment, an increase in the CrI with respect to the BPC due to the hydrolytic scission of the glycosidic bonds occurred. This phenomenon allowed releasing individual crystals and removing the amorphous domains (Lamaming et al. 2015; Lu et al. 2014).Comparing the $\mathrm{CrI}$ of the CNCs with different hydrolysis times, the same behavior as that observed for the gravimetric yield was observed; samples showed an increase in their crystallinity from 30 to $45 \mathrm{~min}$ and then, decreased at $90 \mathrm{~min}$. Therefore, the highest crystallinity is achieved for a hydrolysis time of $45 \mathrm{~min}(88.5 \%)$ whereas the $\mathrm{CNC}_{90}$ showed the lowest CrI (80.8\%). This may be due to the fact that crystalline regions can be partially destroyed with high acid hydrolysis times (Chen et al. 2009; Neto et al. 2013). The CNC crystallinity indexes obtained from PC are similar to those obtained in other agroindustrial and forest residues such as rice straw ( $\mathrm{Lu}$ and Hsieh 2012), spruce bark (Le Normand et al. 2014) or Swedish forest residues (Scots pines and Norway spruce) (Moriana et al. 2016), being higher to those obtained in other lignocellulosic residues such as soy hulls (Neto et al. 2013) or kenaf fibres (Kargarzadeh et al. 2012).

\section{Chemical structure}

The infrared spectra of the PC, APC, BPC and the CNCs with different hydrolysis times are gathered in Fig. 5. Significant changes in the spectra were detected after the different treatments performed, thus demonstrating the effectiveness of each one of them.

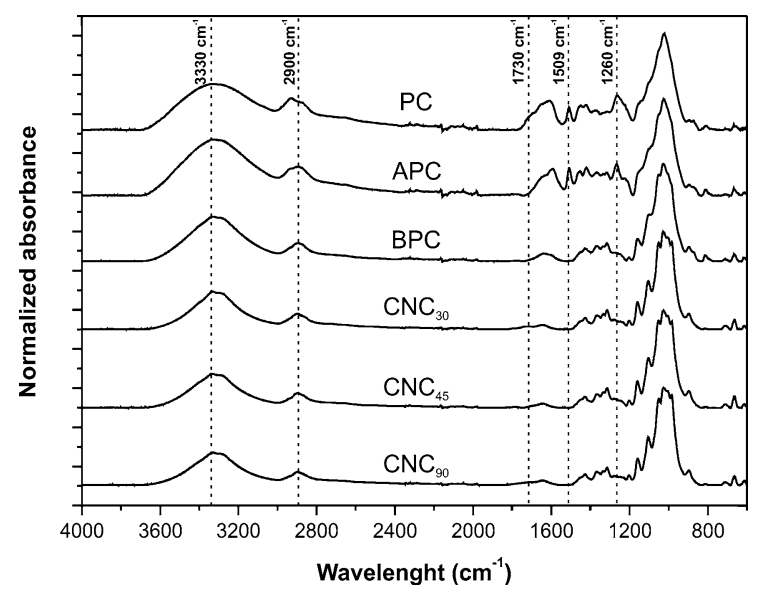

Fig. 5 FTIR spectra of PC, APC, BPC and CNCs with different hydrolysis times 
The peak located around $3330 \mathrm{~cm}^{-1}$, which corresponds to stretching vibrations of $\mathrm{OH}$ groups of cellulose and lignin (Reddy and Rhim 2014), became narrower after the different treatments due to the removal of part of the amorphous components (Le Normand et al. 2014). The peak around $2900 \mathrm{~cm}^{-1}$, which corresponds to the $\mathrm{C}-\mathrm{H}$ stretching of methyl and methylene groups in cellulose (Lamaming et al. 2015), decreased after the alkaline and bleaching treatment. The peak located at $1730 \mathrm{~cm}^{-1}$ attributed to the acetyl and uronic ester groups of the hemicellulose or the ester linkage of carboxylic group of ferulic and p-coumaric acids of lignin and/or hemicelluloses, was also reduced and practically disappeared after bleaching (Chen et al. 2011; Neto et al. 2013). The same was observed for the peak at $1509 \mathrm{~cm}^{-1}$ that corresponds to the $\mathrm{C}-\mathrm{H}$ deformation in methyl, methylene and methoxyl groups of lignin and aromatic $\mathrm{C}=\mathrm{C}$ ring stretching. Similar behavior was observed for the peak located at $1260 \mathrm{~cm}^{-1}$ that corresponds to the $\mathrm{C}-\mathrm{O}-\mathrm{C}$ vibration of acetyl groups in hemicellulose. The absence of these peaks in the BPC and CNC spectra indicates that most of the hemicellulose and lignin was removed, thus leading to a material with high cellulose content as it was shown by chemical analysis.

In CNCs spectra for the different hydrolysis times, it is worthy to note the appearance of a new small peak at $1205 \mathrm{~cm}^{-1}$ related to $\mathrm{S}=\mathrm{O}$ vibration. This can be explained by considering the esterification reaction during the hydrolysis process (Silvério et al. 2013). This peak had a higher intensity as the hydrolysis time increased.

\section{Thermal stability and behaviour}

Thermogravimetric (TG) and first derivative thermogravimetric (DTG) curves for all the studied samples are shown in Fig. 6. Two main mass-loss regions were identified during the thermal decomposition of each sample: a first mass loss $(<6.5 \%)$ between 25 and $150{ }^{\circ} \mathrm{C}$ related to the moisture evaporation and a second region between 150 and $650{ }^{\circ} \mathrm{C}$, where the mass loss was due to thermal degradation of the cellulose, hemicellulose and lignin components. Figure $6 \mathrm{a}, \mathrm{c}$ show that the thermal degradation of PC occurs in three overlapped process between 150 and $650{ }^{\circ} \mathrm{C}$ : the first process was related to the thermal degradation of hemicellulose and pectins and appeared between 200 and $340{ }^{\circ} \mathrm{C}$; the second one was associated with the cellulose degradation (between 340 and $400{ }^{\circ} \mathrm{C}$ ) and showed a well-defined peak at $364{ }^{\circ} \mathrm{C}$ (García-García et al. 2015; Roman and Winter 2004); and the third process related with most of the lignin degradation that appeared as a small shoulder at higher temperatures, between 415 and $525{ }^{\circ} \mathrm{C}$ (Deepa et al. 2015; Le Normand et al. 2014). After the alkaline and bleaching treatment the peak located around $330{ }^{\circ} \mathrm{C}$ and the shoulder at the highest temperatures disappeared due to the removal of hemicellulose and lignin after both chemical treatments (Sheltami et al. 2012). Furthermore, an improvement in thermal stability was observed due to the removal of hemicellulose, which decomposes before lignin and cellulose as a result of the presence of acetyl groups in hemicellulose molecules (Kargarzadeh et al. 2012).

Figure $6 \mathrm{~b}$ shows the thermal degradation behavior of CNCs at different acid hydrolysis times. The acid hydrolysis of the bleached fibres led to a decrease in thermal stability due to the substitution of hydroxyl groups by sulphate groups. First, evaporation of water absorbed by the CNCs occurred, being lower than in the previous steps due to the dehydration of cellulose fibres occurred during the acid hydrolysis (Roman and Winter 2004). Three overlapping processes took place at higher temperatures $\left(>145^{\circ} \mathrm{C}\right)$ : the first one centered at approximately $175^{\circ} \mathrm{C}$ was related to the sulphate groups that catalyze cellulose dehydration; the second peak centred at $220-234{ }^{\circ} \mathrm{C}$ was associated with the breakdown of the more accessible region in the crystal interior; and the last one, with a temperature around $364{ }^{\circ} \mathrm{C}$, was associated with the less accessible crystal interior of the CNCs (Silvério et al. 2013). The thermal degradation behavior of $\mathrm{CNC}_{30}$ was similar to $\mathrm{CNC}_{45}$, however $\mathrm{CNC}_{90}$ displayed a slightly lower thermal stability and a higher second peak due to the higher sulfate content and the degradation of the most labile region in the crystal interior. The residual mass in the CNCs was larger than in the bleached samples and could be explained by the presence of sulfuric acid that promoted the dehydration reactions and acted as a flame retardant (Kim et al. 2001; Roman and Winter 2004). 

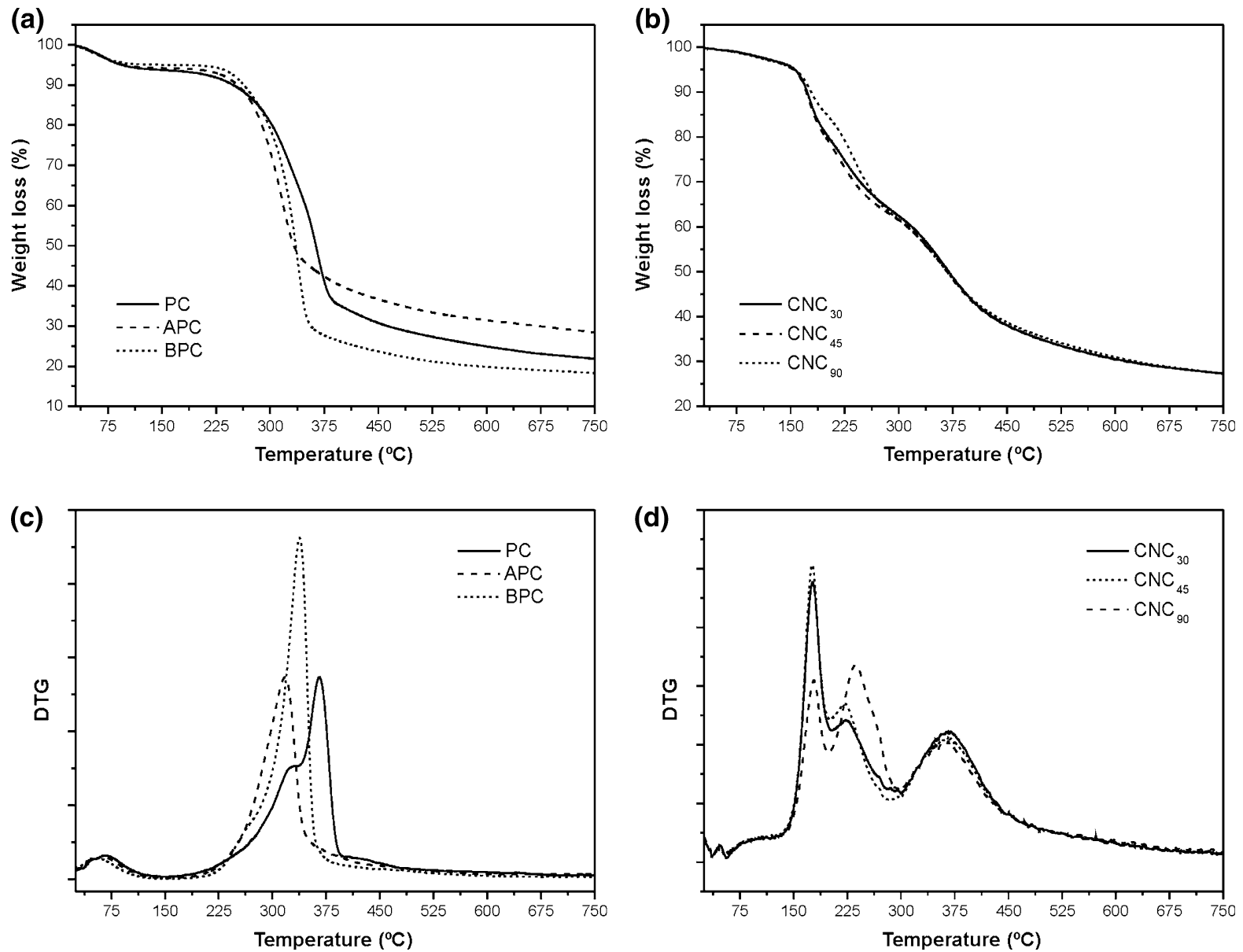

Fig. 6 TGA and DTG curves of a, $\mathbf{c}$ PC, APC, BPC and b, d CNCs with different hydrolysis times

\section{Conclusions}

Cellulose nanocrystals (CNCs) were successfully isolated from pine cones (PC), an abundant and cheap biomass residue. In this work the changes in the chemical composition, morphology, crystallinity and thermal properties of the biomass during the alkaline and bleaching treatment and the influence of the acid hydrolysis time $(30,45$, and $90 \mathrm{~min})$ on the overall yield and on performance of the resulting CNCs have been studied. The progressive removal of lignin and hemicelluloses resulted in extraordinary high cellulosic fibre samples suitable for the subsequent CNCs isolation. The $\mathrm{CNC}_{45}$ showed similar chemical composition, aspect ratio, crystalline size and thermal behavior to those for the $\mathrm{CNC}_{30}$ however, the yield and crystallinity were higher for the $\mathrm{CNC}_{45}$. The
$\mathrm{CNC}_{90}$ exhibited the lowest yield, crystal size, crystallinity index, aspect ratio and thermal stability, indicating that partial destruction of crystalline domains could occur. Therefore, these results show that PC is an effective renewable source for the production of CNCs with an optimal extraction time of around $45 \mathrm{~min}$ during hydrolysis at $45{ }^{\circ} \mathrm{C}$ with $65 \%$ sulfuric acid. The high aspect ratio, the high crystallinity and the good thermal stability of the obtained CNCs show great potential for their use as reinforcement in polymeric composites for different applications.

Acknowledgments This work was supported by the Ministry of Economy and Competitiveness (MINECO) [MAT201459242-C2-1-R]. D. García-García wants to thanks the Spanish Ministry of Education, Culture and Sports for their financial support through an FPU Grant [FPU13/06011]. 
Open Access This article is distributed under the terms of the Creative Commons Attribution 4.0 International License (http:// creativecommons.org/licenses/by/4.0/), which permits unrestricted use, distribution, and reproduction in any medium, provided you give appropriate credit to the original author(s) and the source, provide a link to the Creative Commons license, and indicate if changes were made.

\section{References}

Almendros A, Martín-Lara M, Ronda A, Pérez A, Blázquez G, Calero M (2015) Physico-chemical characterization of pine cone shell and its use as biosorbent and fuel. Bioresour Technol 196:406-412

Beck-Candanedo S, Roman M, Gray DG (2005) Effect of reaction conditions on the properties and behavior of wood cellulose nanocrystal suspensions. Biomacromol 6:1048-1054

Bendahou A, Habibi Y, Kaddami H, Dufresne A (2009) Physico-chemical characterization of palm from phoenix dactylifera-L, preparation of cellulose whiskers and natural rubber-based nanocomposites. J Biobased Mater Bioenergy 3:81-90

Bondeson D, Mathew A, Oksman K (2006) Optimization of the isolation of nanocrystals from microcrystalline cellulose by acid hydrolysis. Cellulose 13:171-180

Brito BS, Pereira FV, Putaux J-L, Jean B (2012) Preparation, morphology and structure of cellulose nanocrystals from bamboo fibers. Cellulose 19:1527-1536

Camarero Espinosa S, Kuhnt T, Foster EJ, Weder C (2013) Isolation of thermally stable cellulose nanocrystals by phosphoric acid hydrolysis. Biomacromol 14:1223-1230

Chen Y, Liu C, Chang PR, Cao X, Anderson DP (2009) Bionanocomposites based on pea starch and cellulose nanowhiskers hydrolyzed from pea hull fibre: effect of hydrolysis time. Carbohydr Polym 76:607-615

Chen W, Yu H, Liu Y, Chen P, Zhang M, Hai Y (2011) Individualization of cellulose nanofibers from wood using high-intensity ultrasonication combined with chemical pretreatments. Carbohydr Polym 83:1804-1811

Cherian BM, Leão AL, de Souza SF, Thomas S, Pothan LA, Kottaisamy M (2010) Isolation of nanocellulose from pineapple leaf fibres by steam explosion. Carbohydr Polym 81:720-725

de Carvalho DM, Sevastyanova O, Penna LS, da Silva BP, Lindström ME, Colodette JL (2015) Assessment of chemical transformations in eucalyptus, sugarcane bagasse and straw during hydrothermal, dilute acid, and alkaline pretreatments. Ind Crops Prod 73:118-126

Deepa B et al (2011) Structure, morphology and thermal characteristics of banana nano fibers obtained by steam explosion. Bioresour Technol 102:1988-1997

Deepa B et al (2015) Utilization of various lignocellulosic biomass for the production of nanocellulose: a comparative study. Cellulose 22:1075-1090

dos Santos RM, Neto WPF, Silvério HA, Martins DF, Dantas NO, Pasquini D (2013) Cellulose nanocrystals from pineapple leaf, a new approach for the reuse of this agrowaste. Ind Crops Prod 50:707-714

Eichhorn SJ et al (2010) current international research into cellulose nanofibres and nanocomposites. J Mater Sci 45:1

García-García D, Carbonell A, Samper M, García-Sanoguera D, Balart R (2015) Green composites based on polypropylene matrix and hydrophobized spend coffee ground (SCG) powder. Compos Part B 78:256-265

Haafiz MM, Eichhorn S, Hassan A, Jawaid M (2013) Isolation and characterization of microcrystalline cellulose from oil palm biomass residue. Carbohydr Polym 93:628-634

Hannuksela T, Tenkanen M, Holmbom B (2002) Sorption of dissolved galactoglucomannans and galactomannans to bleached kraft pulp. Cellulose 9:251-261

Ioelovich M (2012) Optimal conditions for isolation of nanocrystalline cellulose particles. Nanosci Nanotechnol 2:9-13

Iwata T, Indrarti L, Azuma J-I (1998) Affinity of hemicellulose for cellulose produced by acetobacter xylinum. Cellulose 5:215-228

Jiang F, Hsieh Y-L (2013) Chemically and mechanically isolated nanocellulose and their self-assembled structures. Carbohydr Polym 95:32-40

Johar N, Ahmad I, Dufresne A (2012) Extraction, preparation and characterization of cellulose fibres and nanocrystals from rice husk. Ind Crops Prod 37:93-99

Kargarzadeh H, Ahmad I, Abdullah I, Dufresne A, Zainudin SY, Sheltami RM (2012) Effects of hydrolysis conditions on the morphology, crystallinity, and thermal stability of cellulose nanocrystals extracted from kenaf bast fibers. Cellulose 19:855-866

Kim D-Y, Nishiyama Y, Wada M, Kuga S (2001) High-yield carbonization of cellulose by sulfuric acid impregnation. Cellulose 8:29-33

Lamaming J, Hashim R, Sulaiman O, Leh CP, Sugimoto T, Nordin NA (2015) Cellulose nanocrystals isolated from oil palm trunk. Carbohydr Polym 127:202-208

Le Normand M, Moriana R, Ek M (2014) Isolation and characterization of cellulose nanocrystals from spruce bark in a biorefinery perspective. Carbohydr Polym 111:979-987

Li D, Moriana R, Ek M (2016) From forest residues to hydrophobic nanocomposites with high oxygen-barrier properties. Nord Pulp Pap Res J 31:261-269

Loewe V, Navarro-Cerrillo RM, García-Olmo J, Riccioli C, Sánchez-Cuesta R (2017) Discriminant analysis of Mediterranean pine nuts (Pinus pinea L.) from Chilean plantations by near infrared spectroscopy (NIRS). Food Control 73:634-643

Lu P, Hsieh Y-L (2012) Preparation and characterization of cellulose nanocrystals from rice straw. Carbohydr Polym 87:564-573

Lu H, Gui Y, Zheng L, Liu X (2013) Morphological, crystalline, thermal and physicochemical properties of cellulose nanocrystals obtained from sweet potato residue. Food Res Int 50:121-128

Lu Q-L, Tang L-R, Wang S, Huang B, Chen Y-D, Chen X-R (2014) An investigation on the characteristics of cellulose nanocrystals from Pennisetum sinese. Biomass Bioenergy 70:267-272

Luzi F, Fortunati E, Puglia D, Petrucci R, Kenny J, Torre L (2016) Modulation of acid hydrolysis reaction time for the 
extraction of cellulose nanocrystals from Posidonia oceanica leaves. J Renew Mater 4:190-198

Maiti S, Jayaramudu J, Das K, Reddy SM, Sadiku R, Ray SS, Liu D (2013) Preparation and characterization of nanocellulose with new shape from different precursor. Carbohydr Polym 98:562-567

Mariano M, El Kissi N, Dufresne A (2014) Cellulose nanocrystals and related nanocomposites: review of some properties and challenges. J Polym Sci, Part B: Polym Phys 52:791-806

Moriana R, Vilaplana F, Ek M (2015) Forest residues as renewable resources for bio-based polymeric materials and bioenergy: chemical composition, structure and thermal properties. Cellulose 22:3409-3423

Moriana R, Vilaplana F, Ek M (2016) Cellulose nanocrystals from forest residues as reinforcing agents for composites: a study from macro-to nano-dimensions. Carbohydr Polym 139:139-149

Mueller S, Weder C, Foster EJ (2014) Isolation of cellulose nanocrystals from pseudostems of banana plants. RSC Adv 4:907-915

Neto WPF, Silvério HA, Dantas NO, Pasquini D (2013) Extraction and characterization of cellulose nanocrystals from agro-industrial residue-soy hulls. Ind Crops Prod 42:480-488

Özgüven F, Vursavuş K (2005) Some physical, mechanical and aerodynamic properties of pine (Pinus pinea) nuts. J Food Eng 68:191-196

Peng BL, Dhar N, Liu H, Tam K (2011) Chemistry and applications of nanocrystalline cellulose and its derivatives: a nanotechnology perspective. Can $J$ Chem Eng 89:1191-1206

Reddy JP, Rhim J-W (2014) Isolation and characterization of cellulose nanocrystals from garlic skin. Mater Lett 129:20-23
Roman M, Winter WT (2004) Effect of sulfate groups from sulfuric acid hydrolysis on the thermal degradation behavior of bacterial cellulose. Biomacromol 5:1671-1677

Rosli NA, Ahmad I, Abdullah I (2013) Isolation and characterization of cellulose nanocrystals from Agave angustifolia fibre. BioResources 8:1893-1908

Saeman JF, Moore WE, Mitchell RL, Millett MA (1954) Techniques for the determination of pulp constituents by quantitiative paper chromatography. Tappi J 37:336-343

Satyamurthy P, Jain P, Balasubramanya RH, Vigneshwaran N (2011) Preparation and characterization of cellulose nanowhiskers from cotton fibres by controlled microbial hydrolysis. Carbohydr Polym 83:122-129

Segal L, Creely J, Martin A Jr, Conrad C (1959) An empirical method for estimating the degree of crystallinity of native cellulose using the X-ray diffractometer. Text Res J 29:786-794

Sheltami RM, Abdullah I, Ahmad I, Dufresne A, Kargarzadeh H (2012) Extraction of cellulose nanocrystals from mengkuang leaves (Pandanus tectorius). Carbohydr Polym 88:772-779

Silvério HA, Neto WPF, Dantas NO, Pasquini D (2013) Extraction and characterization of cellulose nanocrystals from corncob for application as reinforcing agent in nanocomposites. Ind Crops Prod 44:427-436

Siqueira G, Bras J, Dufresne A (2010) Cellulosic bionanocomposites: a review of preparation, properties and applications. Polymers 2:728-765

Willför S, Sundberg A, Hemming J, Holmbom B (2005) Polysaccharides in some industrially important softwood species. Wood Sci Technol 39:245-257

Wright PJ, Wallis AF (1996) Rapid determination of carbohydrates in hardwoods by high performance anion exchange chromatography. Holzforsch Int J Biol Chem Phys Technol Wood 50:518-524 